\title{
Erratum
}

\section{Mixing of all orders of Lie groups actions}

\author{
Shahar Mozes \\ Department of Mathematic, Hebrew University, Jerusalem 91904, Israel
}

Invent. math. 107, 235-241 (1992)

Professor Stepin has pointed out that in order for the proof of Theorem 1 to work one needs to add to the assumptions of the theorem the requirement that the center of $G$ is finite. This additional change is required to exclude cases where the group $G$ has a nontrivial connected compact central subgroup. In such a case the argument doesn't work as the action of the unipotent element we produce may be non-ergodic. When $G$ has a finite center and is Ad-proper we can choose the open ball $\mathscr{W}$ (see page 238 line -9) so that the element $\left(h_{l+1}, h_{l+2}, \ldots, h_{k}\right)$ has a nontrivial unipotent image under the adjoint representation and hence generates an unbounded subgroup and acts ergodically.

I am grateful to Prof. S.G. Dani, Prof. A. Starkov and Proof. A.M. Stepin for bringing this to my attention. 